\title{
Demand Forecasting in Python: Deep Learning Model Based on LSTM Architecture versus Statistical Models
}

\author{
Ing. Andrea Kolková, Ph.D., Ing. Miroslav Navrátil
}

VSB Technical University of Ostrava, Faculty of Economics, Department of Business Administration, Sokolská třída 33, Ostrava, Czech Republic andrea.kolkova@vsb.cz, miroslav.navratil.st1@vsb.cz

\begin{abstract}
Demand forecasting for business practice is one of the biggest challenges of current business research. However, the discussion on the use of forecasting methods in business is still at the beginning. Forecasting methods are becoming more accurate. Accuracy is often the only criterion for forecasting. In the reality of business practice or management is also influenced by other factors such as runtime, computing demand, but also the knowledge of the manager. The goal of this article is to verify the possibilities demand forecasting using deep learning and statistical methods. Suitable methods are determined on based multi-criteria evaluation. Accuracy according to MSE and MAE, runtime and computing demand and knowledge requirements of the manager were chosen as the criteria. This study used univariate data from an e-commerce entity. It was realized 90-days and 365-days demand forecasting. Statistical methods Seasonal naïve, TBATS, Facebook Prophet and SARIMA was used. These models will be compared with a deep learning model based on recurrent neural network with Long short-term memory (LSTM) layer architecture. The Python code used in all experiments and data is available on GitHub (https://github.com/mrnavrc/demand_forecasting). The results show that all selected methods surpassed the benchmark in their accuracy. However, the differences in the other criteria were large. Models based on deep learning have proven to be the worst on runtime and computing demand. Therefore, they cannot be recommended for business practice. As a best practice model has proven Prophet model developed at Facebook.
\end{abstract}

\section{Introduction and Literature Revue}

At present, demand forecasting is gaining prominence in business economics research. Demand forecasting is now an integral part of corporate management, both operational and strategic management. Today, companies have to forecast a number of variables to be successful in the market, whether it is the number of 
passengers for train seat planning [3], air traffic demand [28], electricity demand [10], demand forecasting in bike-sharing system [31], performing arts [25], forecasting call centre arrivals [23], LCD monitor market [17], or tourism [1]. The analysis of sales and demand is now insufficient and correct forecasting is a key source of information for companies.

This area of business management is also constantly expanding and increasing demands are being placed on the accuracy of forecasting. In this sense, researchers around the world have been developing new and expanding existing methods. Increasing studies are addressing the question whether methods based on machine learning can overcome the existing statistical models. An important contribution in this discussion is a study conducted by Makridakis [19], which has pointed out that machine learning methods cannot itself beat the statistical models in the accuracy of the forecasting. Another study [25], which focuses on the comparison of forecastign methods of COVID-19 active cases, also confirms the abovementioned results. On the other hand, a study [7] conducted at the University of Porto concluded that the study presented by Makridakis is biased with low sample size. Each of the studies used different types of time series for its results.

Statistical methods are still used in business practice, namely, more complex or simple naïve methods or exponential smoothing. The ARIMA method is an often used example for forecasting in tourism in [1]. Already in the last century, statisticians have looked at whether simpler methods are better or worse than more complex ones [15], and at the beginning of this century, Zellner wrote about the need for simplicity of models in practice [32]. In 2018, Makridakis reopened this idea [19]. Time series forecasting is a topic that connects many disciplines [2], [26]. So far, no research has clearly identified the most appropriate method.

The goal of this article is to verify possibilities demand forecasting using deeplearnign and statistical methods. This methods will be used for of the company's 90-days and 365-days demand forecasting.

In this work, the naïve method will be used, it will also serve as a benchmark. Second method used is TBATS based on a study by DeLivera [8]. Another method used is Prophet, developed in practice by a team of experts from Facebook [29], and subsequently verified, for example [23]. Next method which will be used is SARIMAX, which is based on the Box-Jenkins model [6] and is used in pandemic predictions, for example H1N1 [16]. The last model, which will be used, is a deep learning model based on recurrent neural network with Long Short-Term Memory (hereafter only LSTM) layer architecture.

It is also important to mention that the resulting accuracy of the prediction is not necessarily a sign of the quality and usability of the model, but it is necessary to look at other factors, such as the computational demand of the prediction or the financial cost of maintaining such a model in real business operations. Gilliland [9], for example, states in his published discussion that even possibly increased accuracy of prediction, when using highly complex machine learning models, may 
not have its justification in practical use, due to the above-mentioned factors. Among other things, this study aims to point out that future forecasting competitions should include more evaluation criteria and should focus more on real business usability.

\section{Data Description and Methodology}

The selected data set is divided into training data set and test data set, in the ratio 2/3: $1 / 3$. As benchmark are used forecasting by the seasonal naïve forecast. This method are used to evaluate other advanced models.

All forecasts are modelled using Python. Python is being developed as an open source programming language with a number of other packages. It is an interpreted general-purpose project. It is often used for data analytics, but is also used for backend applications. For retrieve data is applied Pandas library, it is developed by Wes McKinney [20].

Pandas operate with the Numpy package, is is array-processing package and fundamental tool for Python scientific research. Used stucture is caled data frame, it can used a table or a two-dimensional array-like structure.

StatsModels and PmdArima (also called PyramidArima) libraries were also used in this study. Statsmodels was used for statistical tests and statistical data exploration.

\subsection{Data Description}

This study uses data from an e-commerce entity. E-shop is focused in the outdoor supplies for professional athletes. This company organizes outdoor training courses, seminars and competitions too. A lot of customers are in the B2B.

Individual daily demand form a data set. It is started at 1st September 2014 and ended 22 ${ }^{\text {th }}$ August 2020. Data set is geted using Google Analytics. Data set contains daily data of demand (number of conversions per day). Data is available at https://github.com/mrnavrc/demand_forecasting. All values was divided with the secret coefficient for trade secret, leading to a reduction in the size of demand in absolute terms. Does not change the trend, cycles and parameters necessary for forecasting. This was confirmed by the decomposition of the data. This decomposition confirmed the growing demand in the observed months in the summer months and in the period before Christmas. The cyclical development did not change throughout the observed period. Historical demand is graphically shown in Figure 1. 


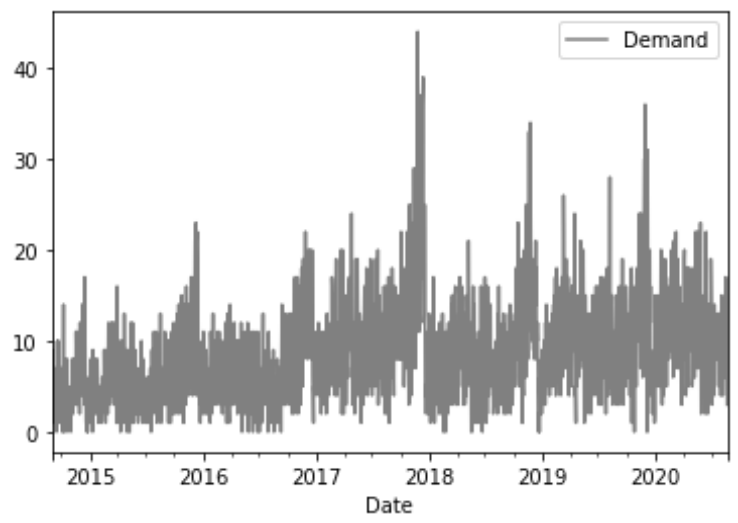

Figure 1

Demand of e-commerce entity from $1^{\text {st }}$ September 2014 to $22^{\text {th }}$ August 2020

Before forecasting, it is necessary to perform a statistical description and analysis of missing values and outliers. Missing values do not appear in the selected data file. There are some outliers. After analysis of the robustness of the selected models we decided let the outliers in the data set. In the histogram in Figure 2 we see the distribution of data around its mean value.

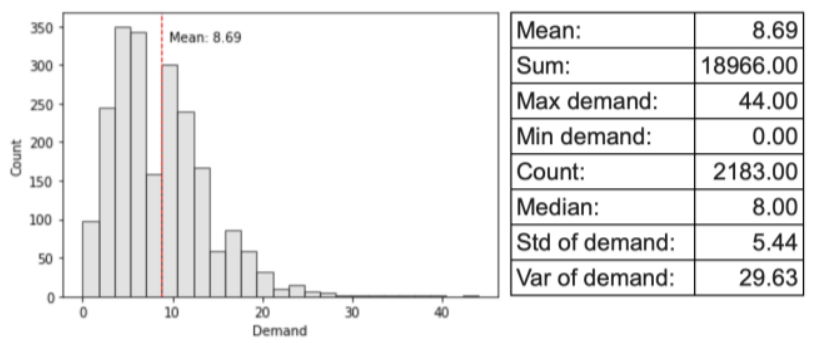

Figure 2

Histogram of demand of e-commerce entity

\subsection{Applied Methods}

The seasonal naïve forecast is based on the idea that the forecast will be equal to the previous period according to the selected time frame of the forecast. It is declarated [22]. This method is based on a simple formula (1).

$\hat{y}_{T+h / T}=y_{T+h-m(k+1)}$, where

$m$ is the seasonal period and $k$ is the integer part of $(h-1) / m$ (i.e. the number of complete years in the forecast period prior to time $T+h$.) 
We make a demand forecast for the outdoor equipment, so can be expected a strong seasonal character. This could mean that this method will be too simple and less suitable for this data [12].

TBATS is acronym from the other models: Trigonometric seasonality, Box-Cox transformation, ARMA errors, Trend and Seasonal components [14]. This designation must be supplemented by appropriate arguments $\left(\varpi, \phi, p, q, m_{l}\right.$, $\left.m_{2} \ldots \ldots \ldots m_{T}\right)$ to define Cox - Box parameters and damping parameters $p$ and $q$ to express the parameter of ARMA models and seasonal periodicity is expressed by arguments $m_{l}, m_{2} \ldots \ldots \ldots m_{T}$. TBATS for modelling prediction automatically considers different models Box-Cox transformation. The best accuracy model will be chosen using Akaike information criterion [22]. The TBATS model is described by the relation (2).

$\varphi \cdot p(L) \cdot \mu(L) \cdot y(\omega) \cdot t=\theta \cdot q(L) \cdot \delta(L) \cdot \varepsilon_{t}$, where

$L$ is the lag operator, $\mu(L)$ is $\operatorname{det}(I-F \cdot L), \delta(L)=w \cdot \operatorname{adj}(I-F \cdot L) g \cdot L+\operatorname{det}(I-208 F \cdot L)$, $\varphi \cdot p(L)$ and $\theta \cdot q(L)$ are polynomials of length $p$ and $q$.

For this forecast we need around 298-461 seconds. TBATS is known for its high computational demand. The model applied grid research and no genetic evolution to set parameters faster.

Prophet is an open source library designed especially for business data forecasting. The prophet was developed by Facebook and has already been used in research [23]. Prophet uses the scikits-learn (SKlearn) library. It's open-source library and contains functions for machine learning and statistical modelling. The model comes from a relationship (3).

$y_{t}=g_{t}+s_{t}+h_{t}+\varepsilon_{t}$, where

$g_{t}$ is the trend function, $s_{t}$ is periodic changes, $h_{t}$ is the effect of the holiday and $\varepsilon_{t}$ is error.

It is possible to import public holidays and other events that affect demand into the Prophet model.

Based on the decomposition, it was found that this e-shop is significantly affected by Christmas, so it is included in the model. Other factors influencing the demand for sporting goods are sporting events such as the championship, Olympics, etc. The event can also be entered in prophet as an interval, many sporting events have an impact on demand many days before and after the event.

Model Prophet accuracy is based on a cross-validation function. In the model we can used accuracy by mean squared error (hereafter onlyMSE), root mean squared 
erron (hereafter only RMSE), mean absolute error (hereafter only MAE), mean absolute percentage error (hereafter only MAPE) and coverage.

The PmdArima library contains the auto_arima function, which was used to calculate the SARIMA model. The model is based on arima models according to the relationship (4).

$\widehat{y_{t}}=c+\emptyset_{1} y_{t-1}+\emptyset_{2} y_{t-2}+\cdots+\emptyset_{p} y_{t-p}+\varepsilon_{t}$

Auto_arima work like grid-search. Defines a model in the form SARIMA $(p, q, d)$. It means, that various sets of $p$ and $q$ (also $P$ and $Q$ for seasonal models) parameters are tested. The company sets whether it wants to select a model based on minimizes the Akaike information criterion (hereafter only AIC) or Bayesian information criterion (hereafter only BIC).

The parameters $p$ and $q$ are selected by the function itself using an automated iterative procedure. Parameter $d$ must be set according to the stationarity tests, which are also part of the package. Prophet, for example, offers the augmented Dickey-Fuller test to test the stationarity and the Canova-Hansen test to test the seasonality.

This approach model parameters making is very time-consuming. It is better the stepwise function use. This stepwise function is definated by the strategy provided by Hyndman and Khandakar [13]. This model is currently considered to be the best model for step-in training process.

After determining the best model according to auto_arima, the SARIMAX function is used. This is part of the Statsmodels library. The result of SARIMAX is a diagnostic overview that can find a mistake or shortcoming in the semiautomatically approach [22]. After the diagnostic verified, a separate demand forecasting can be created.

The next model used is Deep Learning. For a deep learning model demand forecast it is used the Keras library. Keras library is high-level neural network application programming interface (hereafter only API). This library is created in Python. Keras supports convolutional networks, recurrent networks and their combinations too [22].

For demand forecasting by a deep learning using Keras, is difficult to adjust input data. Data set must be preprocessing and it must be converted to tensors. This is done by using the time series generator function. The deep learning model for demand forecasting can be demanding expertise and computing-time. 


\subsection{Multicriteria Approach to Method Evaluation}

As mentioned, the prediction calculations will be performed for a period of 90 days and then for 365 days. The final evaluation is carried out on the basis of a multicriteria approach. The first evaluation criterion will be the degree of accuracy of the model.

Accuracy expresses the basic criterion for evaluating the quality of a model. In business practice, several indicators MAE, RMSE, MAPE and many others are used. The degree of accuracy decreases with the length of the forecast period. Forecasting can never be completely accurate, there is a certain amount of random component in each time series. MAE and MSE will be used in this study.

The mean absolute error (MAE), described relationship (5).

$M A E=\frac{1}{M} \sum_{p=n+1}^{N}\left|y_{p}-\widehat{y_{p}}\right|$

Some authors [18] point to the impossibility of comparing accuracy results between different time series. The MAE result is in units of original demand. In this paper, however, we have a time series of the same parameters, so the use of this criterion is possible.

The second accuracy used is MSE. This indicator is described by the relation (6).

$M S E=\frac{1}{M} \sum_{p=n+1}^{N}\left(y_{p}-\widehat{y_{p}}\right)^{2}$.

In the business situation, where the big error exponentially increases financial costs, is this parameter very important [4].

Another of the parameters of multicriteria comparison are runtime and computing demand. Not only the best accuracy is necessary to select the most suitable forecasting methods. Businesses usually do not have unlimited runtime or computing demand. Sometimes what can be calculated and predicted on campus, with the potential of using supercomputers and excellent computer equipment, is not realistic for business practice. These parameters are therefore crucial for evaluating the effectiveness of the methods used.

The last criterion is the complexity of the researcher's knowledge. Not all companies have top statistical experts and systems engineers. It is therefore necessary that financial and logistics managers be able to interpret or set methods in the company. Today, it is common for methods to be created by companies themselves (such as Google or Facebook), mainly due to the complexity of methods created in academic research. 


\section{Results}

The results of the 90-days demand forecast according to the seasonal naïve method are shown in Figure 3 and Figure 4 for the 365-days demand forecast.

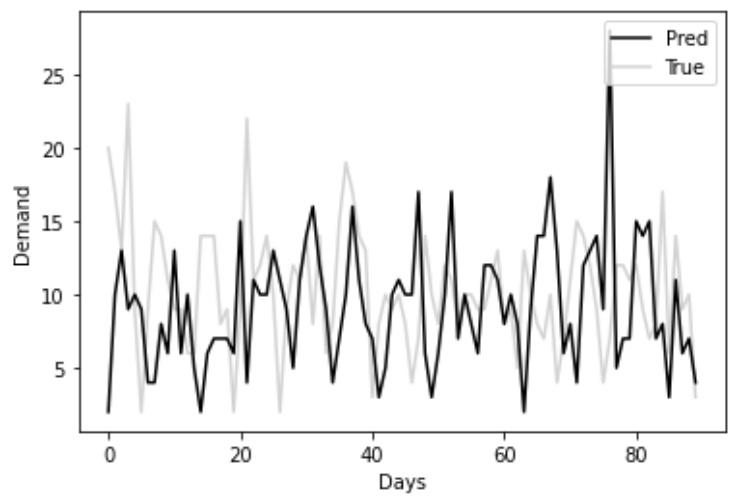

Figure 3

Results of 90-days demand forecasting using seasonal naïve method

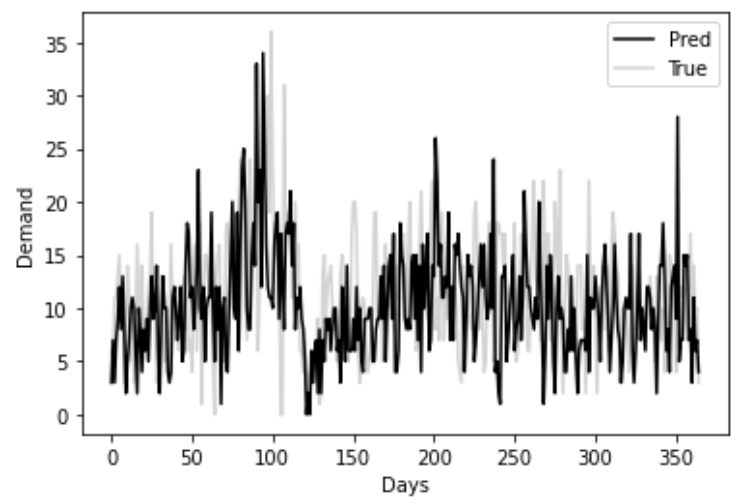

Figure 4

Results of 365-days demand forecasting using seasonal naïve method

Demand forecasting in Figure 4 shows, it will be very difficult to overcome the seasonal naïve forecast. Since the last period the demand changed only a little on data set and the seasonal patterns in demand stay constant. This is a problem with a change of weather in different seasons. This could mean a significant demand shift. Subsequently, there could be insufficient or, on the contrary, excessive supply and subsequent logistical problems.

When forecasting according to the TBATS method, an estimator including settings to handle multi-seasonality was selected based on the Akaike information 
criterion, estimator $=7,365$. Annual and weekly seasonality are including in estimator.

The results of this forecast are shown in Figures 5 and 6. Figure 5 expresses a 90day demand forecast, Figure 6 for 365-days demand forecast.

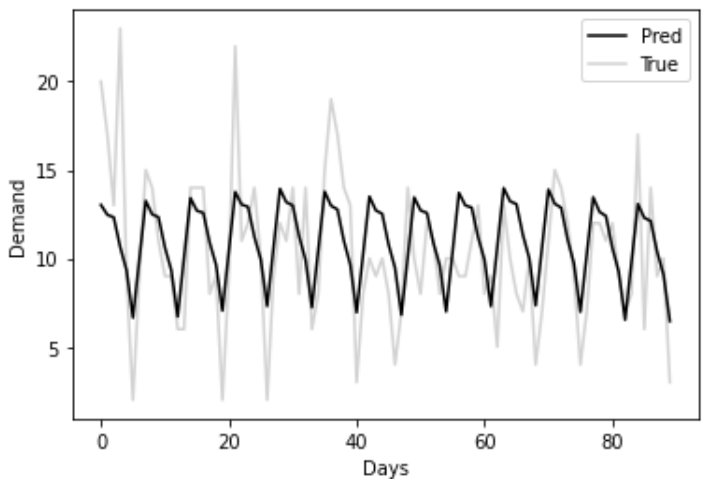

Figure 5

Results of 90-days demand forecasting using TBATS method

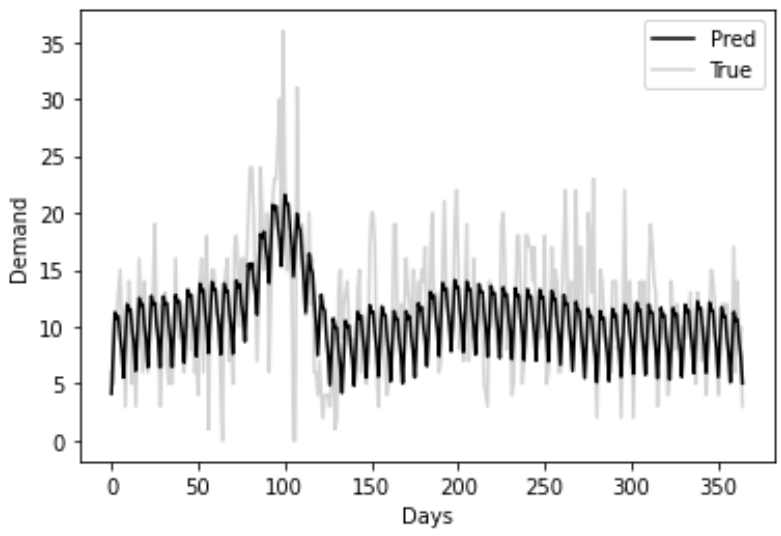

Figure 6

Results of 365-days demand forecasting using TBATS method

Based on the Prophet model, 90 and 365-days demand forecasts were again performed. The results are shown in Figures 7 and 8. 


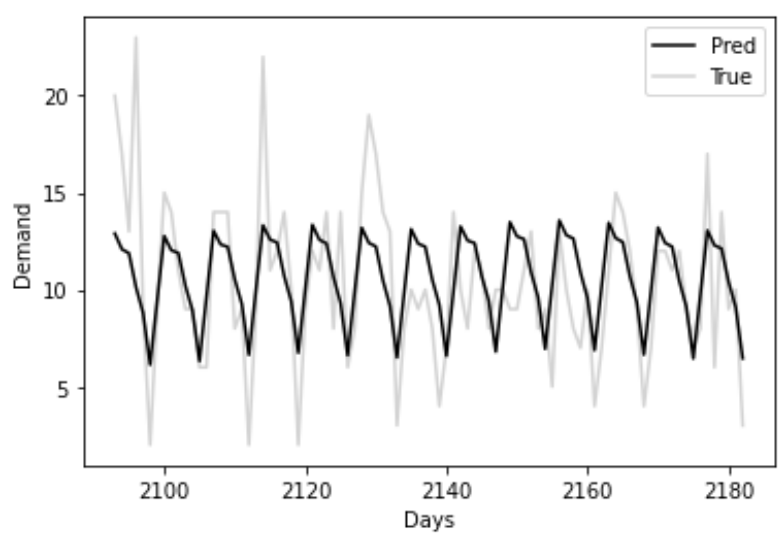

Figure 7

Results of 90-days demand forecasting using Prophet method

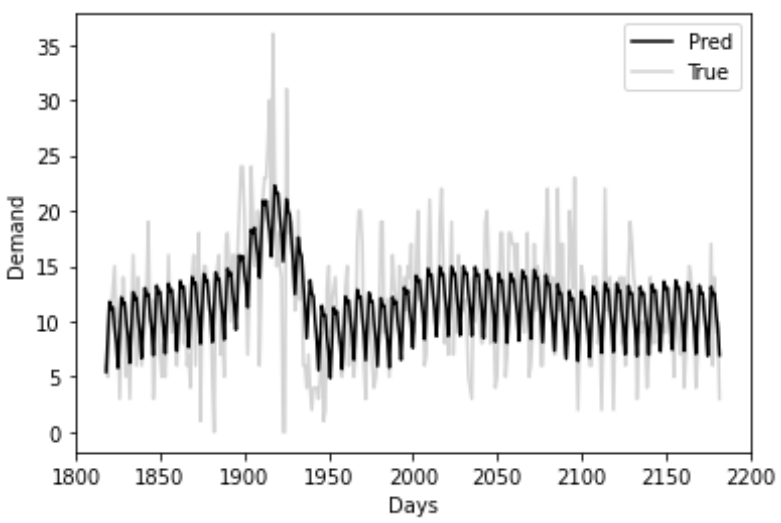

Figure 8

Results of 365-days demand forecasting using Prophet method

According to the methodology, the SARIMAX model was created. When evaluating the models, a model with parameters for 90 days,

$\operatorname{SARIMAX}(0,1,1) x(1,1,1,7)$

was selected by an automated process. For 365-days forecast,

$\operatorname{SARIMAX}(0,0,2) \times(0,1,1,7)$.

The resulting forecast is shown in Figures 9 and 10. 


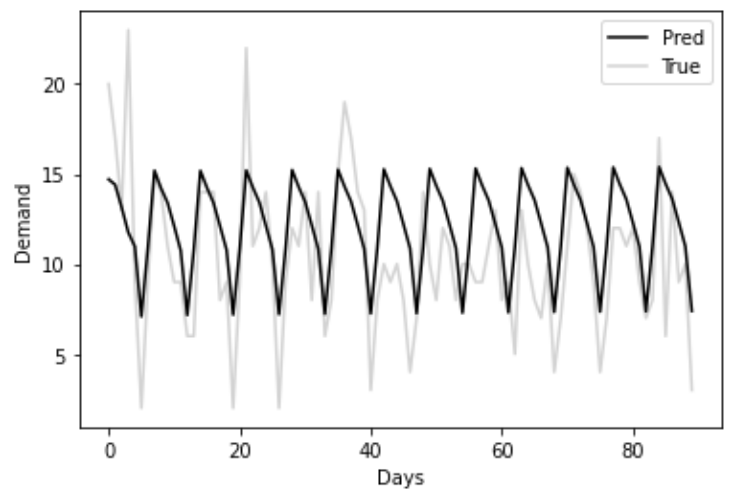

Figure 9

Results of 90-days demand forecasting using SARIMAX method

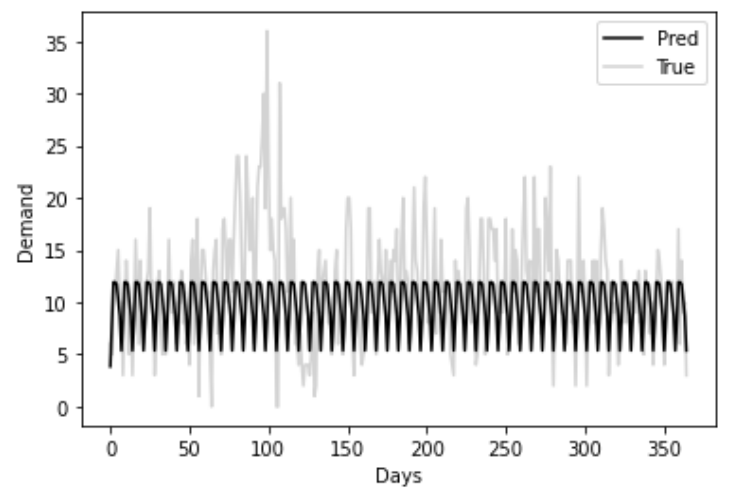

Figure 10

Results of 365-days demand forecasting using SARIMAX method

For Deep learning models, it is necessary to set more parameters. The calculation is therefore relatively demanding on the researcher's experience. Two models were chosen to solve the forecast using deep learning. The model A is based on shallow layers. Model B contains multiple hidden layer, so it is a deep neural network model to be able to compare both deep learning models. The only thing that will change will be the number of layers, and at the same time, both models must set around 360,000 parameters. This model should include more information, and be better to forecast demand curve [30]. This has not been confirmed. The model parameters are listed in Table 1. 
Table 1

Parametrs Deep Learning models

\begin{tabular}{|c|c|c|c|c|c|c|}
\hline \multicolumn{7}{|c|}{ Shallow Layers (model A) } \\
\hline batch size & hidden layers & neurons & dropout & optimizer & epochs \\
\hline 10 & 1 & 300 & 0.1 & Adam & 100 \\
\hline \multicolumn{7}{|c|}{ Deep Layers (model B) } \\
\hline batch size & hidden layers & neurons & dropout & optimizer & epochs \\
\hline 10 & 3 & 405 & 0.1 & Adam & 100 & \\
\hline \multicolumn{7}{|c|}{ Shallow Layers (model A) } \\
\hline batch size & hidden layers & neurons & dropout & optimizer & epochs \\
\hline 10 & 1 & 30 & 0.1 & Adam & 100 \\
\hline batch size & hidden layers & neurons & dropout & optimizer & epochs \\
\hline 10 & 2 & 80 & 0.1 & Adam & 100 & \\
\hline
\end{tabular}

The result of the deep learning MODEL A - shallow layer model for the 90-days forecast is shown in Figure 11 and of the deep learning MODEL B - deep layer in Figure 12.

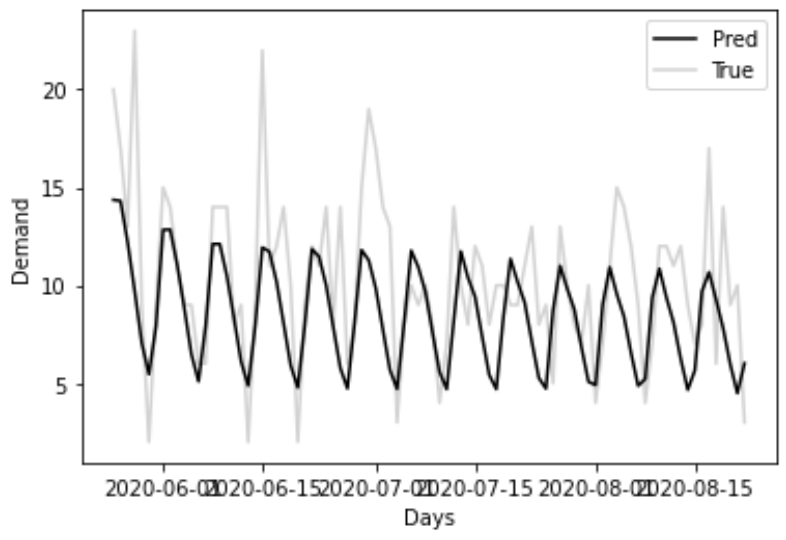

Figure 11

Results of 90-days demand forecasting using Deep learning MODEL A - shallow layer method 


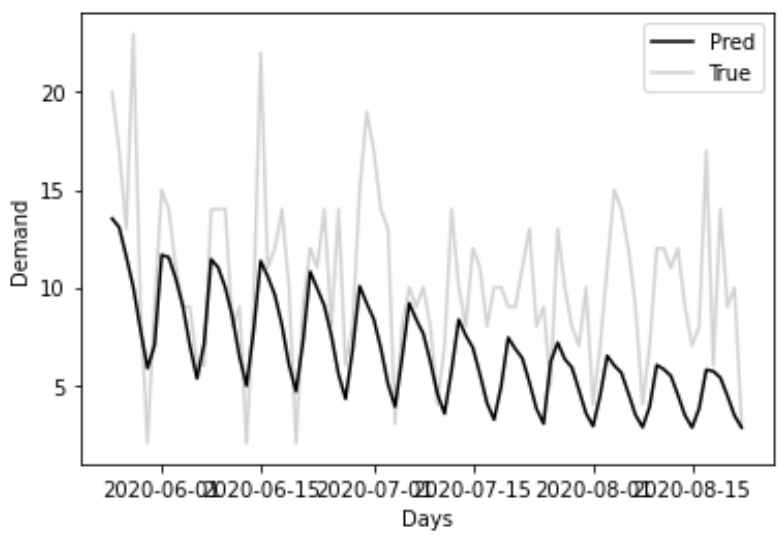

Figure 12

Results of 90-days demand forecast using deep learning MODEL B - deep layer method

The results of demand forecasting show that deep neural networks are not suitable for a simple univariete time series. Those models can be better for complex forecasting problems with mutlivariate data. The calculation of this model is even on an univariate date too time consuming, for this particular forecast the total computer time was 56 minutes. Therefore, it is not effective to continue optimizing for a possible improvement in accuracy.

For 365-days forecast and shallow layer, the results are shown in Figure 13 and deep layers in Figure 14.

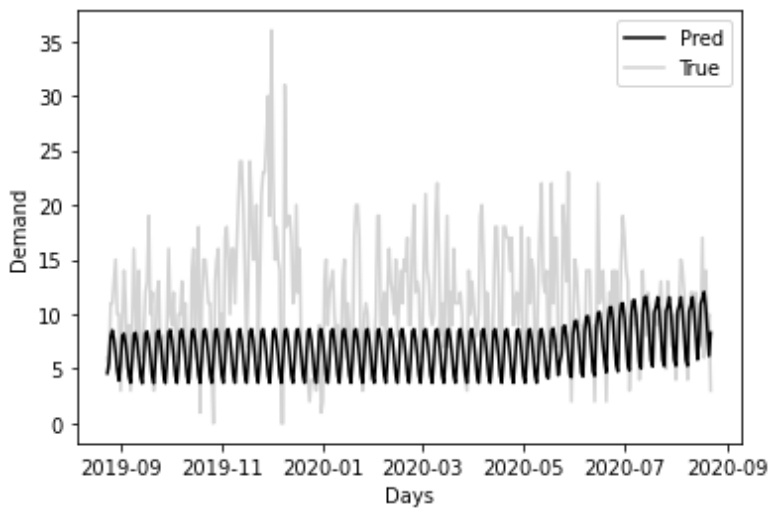

Figure 13

Results of Deep learning MODEL A - shallow layer method for 365-days demand forecast 


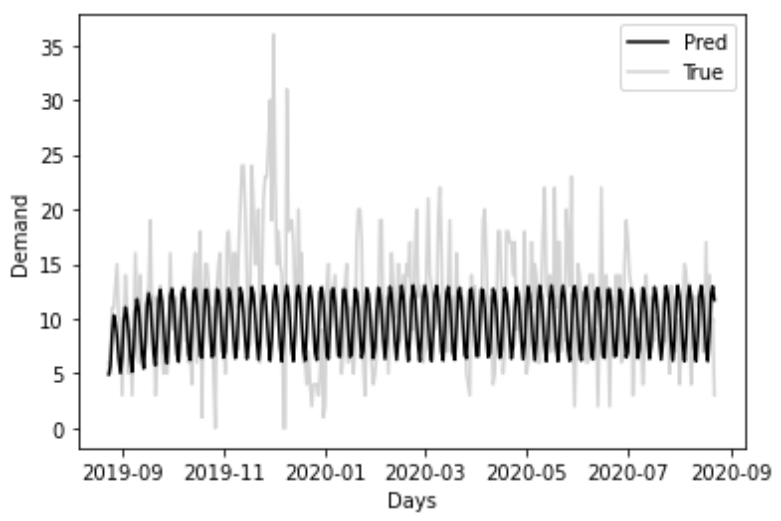

Figure 14

Results of Deep learning MODEL B - deep layer method for 365-days demand forecast

All models were evaluated on the basis of a multicriteria approach. Those criteria were the evaluation of MAE, MSE, runtime and computing demand and the knowledge and experience requirements of the researcher. All models based on the evaluation of accuracy (MAE, MSE) exceeded the benchmark of seasonal naive method. The individual criteria were evaluated in the order they received in the given criterion. Subsequently, the sum of the results of the individual orders was performed (lower is better).

As already mentioned, 5 criteria were evaluated, namely MAE, MSE, runtime, computing demand and researcher's expirence, namely on 90-days demand forecast and 365-days demand forecast.

In Table 2 are the results of multicriterial approach, which was applied in this study, based 90-days demand forecast. The Prophet model is under these criteria the best.

Table 2

Results of multicriteria evaluation models for 90-days demand forecast

\begin{tabular}{|c|r|r|r|r|l|l|l|r|r|r|}
\hline \multicolumn{2}{|c|}{ Model } & M & & & & & & $\begin{array}{l}\text { Computing } \\
\text { demand - } \\
\text { rank }\end{array}$ & $\begin{array}{l}\text { Researcher's } \\
\text { experience - } \\
\text { rank }\end{array}$ & $\begin{array}{l}\text { Total } \\
\text { Rank }\end{array}$ \\
\hline Reasonal naive model & 4.81 & $\mathbf{5}$ & 40.3 & $\mathbf{6}$ & $0.03 \mathrm{~s}$ & $\mathbf{1}$ & $\mathbf{1}$ & $\mathbf{1}$ & 14 \\
\hline \multicolumn{2}{|c|}{ TBATS } & 2.59 & $\mathbf{2}$ & 10.84 & $\mathbf{2}$ & $461.91 \mathrm{~s}$ & $\mathbf{4}$ & $\mathbf{4}$ & $\mathbf{2}$ & 14 \\
\hline \multicolumn{2}{|c|}{ Prophet } & 2.51 & $\mathbf{1}$ & 10.63 & $\mathbf{1}$ & $5.18 \mathrm{~s}$ & $\mathbf{2}$ & $\mathbf{2}$ & $\mathbf{2}$ & 8 \\
\hline \multicolumn{2}{|c|}{ SARIMA } & 2.94 & $\mathbf{3}$ & 12.98 & $\mathbf{3}$ & $17.94 \mathrm{~s}$ & $\mathbf{3}$ & $\mathbf{3}$ & $\mathbf{2}$ & 14 \\
\hline $\begin{array}{c}\text { Deep } \\
\text { Learning }\end{array}$ & Shallow Layers & 2.94 & $\mathbf{3}$ & 14.6 & $\mathbf{4}$ & $2550.10 \mathrm{~s}$ & $\mathbf{5}$ & $\mathbf{5}$ & $\mathbf{3}$ & 20 \\
\cline { 2 - 11 } & Deep Layers & 3.94 & $\mathbf{4}$ & 24.09 & $\mathbf{5}$ & $3400.00 \mathrm{~s}$ & $\mathbf{6}$ & $\mathbf{6}$ & $\mathbf{3}$ & 24 \\
\hline
\end{tabular}


Prophet also showed great results in the 365-days demand forecast, as shown in Table 3. In addition, the practical use of the Prophet model is supported by the conclusions of the already mentioned study, which suggested that information on promotions, holidays and events is very useful. This information can be very easily implemented into Prophet, so it further increases the possibility of use in practice. On the other hand, the exogenous time series that must be predicted (weather, macroeconomic data, etc.) did not bring any significant benefits [5]. And this is an area that needs more testing.

The worst results by this conditions achieved Deep learning model based on shallow layers and deep layers too. Especially for the 365-days demand forecast models using recurrent neural network showed the worst results, see Table 3. By 365-days demand forecasting is the best model Prophet, as well as by 90 -days demand forecasting.

Table 3

Results of multicriteria evaluation models for 365-days demand forecast

\begin{tabular}{|c|c|c|c|c|c|c|c|c|c|c|}
\hline \multicolumn{11}{|c|}{ 365-days demand forecast } \\
\hline & Model & MAE & Rank & MSE & Rank & Runtime & Rank & $\begin{array}{l}\text { Computing } \\
\text { demand - } \\
\text { rank }\end{array}$ & $\begin{array}{l}\text { Researcher's } \\
\text { experience - } \\
\text { rank }\end{array}$ & $\begin{array}{l}\text { Total } \\
\text { Rank }\end{array}$ \\
\hline Seasona & lal naive model & 5.38 & 6 & 48.5 & 6 & $0.04 \mathrm{~s}$ & 1 & 1 & $\mathbf{1}$ & 15 \\
\hline & TBATS & 3.2 & 2 & 17.99 & 2 & $298.28 \mathrm{~s}$ & 4 & 4 & 2 & 12 \\
\hline & Prophet & 3.22 & 1 & 17.69 & 1 & $5.39 \mathrm{~s}$ & 2 & 2 & 2 & $\varepsilon$ \\
\hline & ARIMA & 3.64 & 3 & 24.84 & 3 & $88.62 \mathrm{~s}$ & 3 & 3 & 2 & 1 \\
\hline \multirow{2}{*}{\begin{tabular}{|c|} 
Deep \\
Learning
\end{tabular}} & Shallow Layers & 5.27 & 5 & 46.33 & 5 & $1600.00 \mathrm{~s}$ & 5 & 5 & 3 & 2 \\
\hline & Deep Layers & 4.85 & 4 & 37.5 & 4 & $3000.00 \mathrm{~s}$ & 6 & 6 & 3 & 23 \\
\hline
\end{tabular}

\section{Conclusions}

The goal of this article was to verify the possibility demand forecasting using deep learning and statistical methods. Suitable methods are determined based on multicriteria evaluation. Accuracy according to MSE and MAE, runtime, computing demand and knowledge requirements of the manager were chosen as criteria. In this study was counted the forecast based on a deep learning method and based on statistical methods. Was realized by the e-commerce company 90days and 365- days demand forecasting. Statistical methods Seasonal naïve, TBATS, Facebook Prophet and SARIMA were used. These models will be compared with a deep learning model based on recurrent neural network with Long short-term memory (LSTM) layer architecture.

The seasonal naïve model was chosen as a benchmark in this study. Visual presentation of the prediction indicated that it would be difficult to overcome this benchmark in univariate data. However, based on accuracy measures, the seasonal naïve model was eventually surpassed by all models. 
The Propet model appears to be the most advantageous model for a company in the category of small and medium-sized enterprises. His demands for runtime, computing demand and researcher's experience were at a very good level. These parameters were surpassed only by the seasonal naive method, which had the worst accuracy. Prophet accuracy was the best for both the 90-days and 365-days demand forecast models. Therefore, the resulting multi-criteria comparison rated Prophet as the best.

The TBATS model according to the results can be called the second best. Its accuracy is comparable to the best model. However, TBATS is generally more computationally intensive and therefore its practical business use is likely to have its limitations.

Furthermore, this demand forecast indicate that SARIMA dont capture multiseasonality and daily data. This is declareted other researches too [11]. SARIMA is also not suitable for practical use for demand forecasting due to its high degree of expertise of the processor and the manager working with the results of the model. Even though the resulting forecasting error is not so large, SARIMA cannot be recommended for wider business use.

The deep learning models exceeded the benchmark in the evaluation of accuracy measures and from this point of view they seemed applicable. However, their runtime and the need for high expertise put them in the last place. Small and medium-sized companies, such as the analysed one, usually do not have such computing capacity or relevant knowledge. The deep learning model can be improved. One option may be, setting better parameters as a study, which analysed Kaggles forecasting competitions, pointed out that a key to good performance of many machine learning models is the appropriate selection of hyperparameters and features [5]. However, this is not the subject of this study, because this study focuses only on comparing models in the basic setup without further optimization.

It may also indicated that machine learning models are not suitable for practical use in small and medium-sized companies due to high demands on finance and knowledge.

This is in in accordance with the results of the previous study by Makridakis [19]. His study declareted that on univariate time series classic statistical models is better to use than machine learning models.

Thus, this study supports the conclusion of a study that analysed Wikipedia web traffic and found that one of the weaknesses of recurrent neural networks is in modelling long-term dependencies such as yearly seasonality [5]. However, large multinational companies and companies engaged in professional forecasting invest a lot of money in the development of demand forecasting models. Therefore, neural networks may not be completely excluded now, their further development and possible further use for univariete data can be expected. 
For smaller companies, however, this is not feasible. It is possible for them to use models only in the basic settings. Therefore deep learning models can be very helpful in predicting complex time series data with many exogenous inputs. Especially with the simultaneous use of statistical methods. After all, this was also confirmed by the M4 forecasting competition, where the best models include those based on a hybrid approach. In addition, another study suggests that the requirement for a successful use of machine learning models is cross-learning. That means using many time series to train a single model [27].

\section{Acknowledgement}

This work was supported by the Czech Science Foundation (GACR Project GA18-13951S).

\section{References}

[1] Andreeski, C. and Mechakaroska, D. Modelling, Forecasting and Testing Decisions for Seasonal Time Series in Tourism. Acta Polytechnica Hungarica, 2020, 17(10), pp. 149-171

[2] Farooq, U., Gu, J., Balas, V., Abbas, G., Asad, M. and Balas, M. A Hybrid Time Series Forecasting Model for Disturbance Storm Time Index using a Competitive Brain Emotional Neural Network and Neo-Fuzzy Neurons. Acta Polytechica Hungarica, 2019, 16(4), pp. 213-229

[3] Banerjee, N., Morton, A. and Akartunali, K. Passenger Demand Forecasting in Scheduled Transportation. European Journal of Operational Research, 2020, 286(3), pp. 797-810

[4] Barbieri, N. Approaches to Recommendations. 2014, California: Morgan and Claypool Publishers

[5] Bojer, C. and Meldgaard, J. Learnings from Kaggle's Forecasting Competitions. 2020, Work. Paper

[6] Box, G. E. P., Jenkins, G. M. and Reinsel, G. C. Time Series Analysis: Forecasting and Control. 1976, New York: John Wiley and Sons Inc.

[7] Cerqueira, V., Torgo, L. and Soares, C. Machine Learning vs. Statistical Methods for Time Series Forecasting: Size Matters. Machine Learning. ArXiv, 2019, abs/1909.13316

[8] DeLivera, A., Hyndman, R. J. and Snydera, R. D. Forecasting Time Series with Complex Seasonal Patterns Using Exponential Smoothing. Journal of the American Statistical Association, 2011, 106(496), pp. 1513-1527

[9] Gilliland, M. The Value Added by Machine Learning Approaches in Forecasting. International Journal of Forecasting, 2019, 36(1), pp. 161-166

[10] He, Y. X., Wang, M. Y., Guang, F. T. and Zhao, W. B. Research on the Method of Electricity Demand Analysis and Forecasting: The Case Of China. Electric Power Systems Research, 2020, 187 
[11] Hyndman, R. Forecasting with Long Seasonal Periods. 2020, available at: https://robjhyndman.com/hyndsight/longseasonality/

[12] Hyndman, R. and Athanasopoulos, G. Forecasting, Principles and Practise. 2018, Middletown: Otext.

[13] Hyndman, R. and Khandakar, Y. Automatic Time Series Forecasting: The forecast Package for R. Journal of Statistical Software, 2008, 27(3)

[14] Hochreiter, S. and Schmidhuber, J. Long Short-term Memory. Neural computation, 1997, 9 (8), 1735-1780

[15] Chatfield, C. Simple is Best? International Journal of Forecasting, 1986, 2, pp. 401-402

[16] Choi S. B. and Ahn I. Forecasting Seasonal Influenza-Like Illness in South Korea after 2 And 30weeks Using Google Trends and Influenza Data From Argentina. PLoS ONE, 2020, 15(7)

[17] Lo, S. L, Wang, F. K. and Lin, J. T. Forecasting for the LCD Monitor Market. Journal of Forecasting, 2008, 27(4), pp. 341-356

[18] Macurová, P., Klabusayová, N. and Tvrdoň, L. Logistika, $2^{\text {nd }}$ edition, 2018, Ostrava: VŠB-TU Ostrava

[19] Makridakis S, Spiliotis E. and Assimakopoulos V. Statistical and Machine Learning Forecasting Methods: Concerns and Ways Forward. PLOS ONE, 2018, 13(3)

[20] McKinney, W. Python for Data Analysis Book,: Data Wrangling with Pandas, NumPy, and IPython, $2^{\text {nd }}$ edition, 2017, Sebastopol: O'Reilly Media, Inc.

[21] Millan-Ruiz, D. and Hidalgo, J. I. Forecasting Call Centre Arrivals. Journal of Forecasting, 2013, 32(7), pp. 628-638

[22] Navrátil, M. (2019) Comparison of Statistical Models with Deep Learning in Business Practice. Diploma thesis, VŠB -TU Ostrava, Department of Business Administration

[23] Navrátil, M. and Kolková, A. Decomposition and Forecasting Time Series in the Business Economy Using Prophet Forecasting Model. Central European Business Review, 2019, 8(4), pp. 26-39

[24] Ozhegov, E. M. and Ozhegova, A. Regression Tree Model for Prediction of Demand with Heterogeneity and Censorship. Journal of Forecasting, 2020, 39, pp. 489-500

[25] Papastefanopoulos, V., Linardatos, P. and Kotsiantis, S. COVID-19: A Comparison of Time Series Methods to Forecast Percentage of Active Cases per Population. Applied Sciences, 2020, 10(11) 
[26] Parra, J., Fuentes, O., Anthony, E. and Kreinovich, V. Use of Machine Learning to Analyze and -Hopefully - Predict Volcano Activity. Acta Polytechnica Hungarica, 2020, 14(3), pp. 209-221

[27] Smyl, S. A Hybrid Method of Exponential Smoothing and Recurrent Neural Networks for Time Series Forecasting. International Journal of Forecasting, 2019, 36(1), pp. 75-85

[28] Solvoll, G., Mathisen, T. and Welde, M. Forecasting Air Traffic Demand for Major Infrastructure Changes. Research in Transportation Economics, 2020, 82

[29] Taylor, S. and Letham, B. Forecasting at Scale. The American Statistician, 2018, 72, pp. 37-45

[30] Wei, A. and Wei, J. Deep Learning Essentials: Your Hands-On Guide to the Fundamentals of Deep Learning and Neural Network Modelling. 2018, Birmingham: Pack Publishing

[31] Yang, Y. X., Heppenstall, A., Turner, A. and Comber, A. Using Graph Structural Information About Flows to Enhance Short-Term Demand Prediction in Bike-Sharing Systems. Computers Environment and Urban Systems, 2020, 83

[32] Zellner, A. Keep it sophisticatedly simple. Simplicity, Inference and Modelling: Keep it Sophisticatedly Simple, 2001, Cambridge: Cambridge University Press, pp. 242-262 